\title{
Médiévales
}

Langues, Textes, Histoire

62 | printemps 2012

Hagiographie et réforme dans l'Occident latin

\section{Les Dialogues de Grégoire le Grand et leur postérité : une certaine idée de la réforme?}

Gregory the Great's Dialogues and their Legacy: a Certain Idea of what a Reform can be?

\section{Bruno Dumézil et Sylvie Joye}

\section{OpenEdition}

Journals

\section{Édition électronique}

URL : https://journals.openedition.org/medievales/6619

DOI : 10.4000/medievales.6619

ISSN : $1777-5892$

Éditeur

Presses universitaires de Vincennes

Édition imprimée

Date de publication : 2 juin 2012

Pagination : 13-31

ISBN : 978-2-84292-346-4

ISSN : 0751-2708

\section{Référence électronique}

Bruno Dumézil et Sylvie Joye, « Les Dialogues de Grégoire le Grand et leur postérité : une certaine idée de la réforme? », Médiévales [En ligne], 62 | printemps 2012, mis en ligne le 28 juin 2012, consulté le 23 avril 2022. URL : http://journals.openedition.org/medievales/6619; DOl : https://doi.org/10.4000/ medievales.6619 
Médiévales 62, printemps 2012, p. 13-32

Bruno DUMÉZIL et Sylvie JOYE

\section{LES DIALOGUES DE GRÉGOIRE LE GRAND ET LEUR POSTÉRITÉ: UNE CERTAINE IDÉE DE LA RÉFORME?}

La figure de Grégoire le Grand est essentielle, tant pour l'histoire de l'Église que par l'étendue de son œuvre ou par son influence et sa renommée dans les siècles suivants ${ }^{1}$. Pape, vénéré comme un saint ${ }^{2}$, il a rédigé une œuvre littéraire qui marque un tournant dans le genre hagiographique en pleine constitution ${ }^{3}$, œuvre connue sous le nom de Dialogues ${ }^{4}$. Le caractère extraordinaire de cet ouvrage a amené certains chercheurs à douter de son attribution au pontife de la fin $\mathrm{du} \mathrm{VI}{ }^{\mathrm{e}}$ siècle, mais celle-ci est aujourd'hui presque universellement admise ${ }^{5}$. Les Dialogues sont donc désormais étudiés en relation étroite avec le reste de l'œuvre de Grégoire: une œuvre de théologien, de prêcheur et de moraliste

1. En particulier C. Dagens, Saint Grégoire le Grand.Culture et expérience chrétiennes, Paris, 1977 (p. 247-345 à propos de la conversion).

2. Philippe Bernard ( Sanctus Gregorius Papa. Le Missale Gothicum et le culte du pape Grégoire le Grand dans la Gaule de la fin du VII e siècle », Francia, 32/1 [2005], p. 167-183) a montré comment le culte de Grégoire se développe essentiellement, au moins pour la Gaule, à partir du milieu du VIII ${ }^{\mathrm{e}}$ siècle.

3. S. BoEsch Gajano, «La memoria della santità: Gregorio Magno autore e oggetto di scritture agiografiche», dans Gregorio Magno nel XIV centenario della morte. Convegno internazionale. Roma, 22-25 ottobre 2003, Rome, 2004, p. 321 sq.; A. Degl'Innocenti, A. De Prisco et E. Paoli éd., Gregorio Magno et l'agiografia fra IV e VII secolo (Vérone, décembre 2004), Florence, 2007 (Archivum Gregorianum, 12).

4. Grégoire le Grand, Dialogues, éd. A. de Vogüé, trad. P. Antin, Paris, 1978-1980 (3 vol.).

5. F. CLARK, The Gregorian Dialogues and the Origins of Benedictine Monasticism, Leyde, 2003. Ce même auteur avait avancé dans un ouvrage précédent l'hypothèse selon laquelle les Dialogues seraient une forgerie: The Pseudo-Gregorian Dialogues, Leyde, 1987. La paternité des Dialogues semble cependant bien devoir être attribuée à Grégoire, comme l'indiquent les travaux de Paul Meyvaert et Adalbert de Vogüé. 
renommé, mais aussi l'œuvre d'un administrateur talentueux. À une époque où l'Église n'est pas unifiée à la façon de celle de son homonyme Grégoire VII, Grégoire Ir le Grand s'active pour convertir les marges de l'Occident chrétien tout en transmettant ce que l'on peut appeler les prémices d'une réforme de l’Église, guère appuyée ou diffusée, tandis que Rome n'avait pas pris cette place centrale qu'on lui connaîtra quelques siècles plus tard. Si les Lettres de Grégoire, conservées dans le célèbre Registre qui a consigné des epistolae envoyées ex officio par le pape sous son pontificat, nous montrent des efforts de mise en ordre de la société chrétienne par des admonestations et des conseils ad hominem, il ne s'agit pas de réforme visant à un retour à l'ancien monde, à des règles de l'âge d'or. La réforme promue par Grégoire ne se définit pas alors par un retour historique à une institution passée et parfaite : elle se construit en s'appuyant sur les règles existantes, en insistant sur un ensemble normatif en construction, en cherchant à redresser ce qui défaille et ce qui plie ${ }^{6}$. L'hagiographie est bien propre à évoquer ces changements, dont la radicalité est exprimée par la multiplicité et l'importance des miracles: leur abondance a longtemps jeté un doute pour les érudits sur la valeur des Dialogues; il conviendrait de considérer plutôt ces miracles comme les armes de Grégoire pour réformer les communautés chrétiennes.

La réforme selon Grégoire, à la frontière entre l'Antiquité et le Moyen Âge, correspond à une volonté d'organisation et de correction, de remise en ordre, sans référence explicite ou régulière, dans les Dialogues eux-mêmes, à un retour à une forme de communauté primitive idéale. Il s'agit davantage d'une remise en ordre morale du monde laïque, sur le modèle de ce monde monastique qui est, lui, le modèle très actuel dans lequel Grégoire s'est épanoui. Grégoire promeut ce modèle monastique, à tel point qu'il transforme son palais du Caelius en une communauté monastique et qu'il fonde six monastères en Sicile avec les biens et les domaines familiaux. Le miracle de la vie monastique: voilà l'objectif de la réforme selon Grégoire. Lui-même se sent transformé, «réformé » dans un des miracles qu'il décrit et qui le concerne, lorsqu'il explique que la prière d'un de ses coreligionnaires le change intérieurement ${ }^{7}$. Perclus de douleurs et ne pouvant jeûner, il confie une demande à un frère, Eleuthère, dont l'efficacité de la prière fait prendre conscience de lui-même au futur pape: Grégoire ne se

6. En ce sens, la définition de la réforme mise en œuvre par Grégoire s'approche davantage de celle proposée par le Trésor de la Langue Française: la «réforme» est une «correction, [un] changement profond, [une] transformation, par des moyens conformes aux règles existantes, de quelque chose en vue de le réorganiser, d'améliorer son fonctionnement, ses résultats » plutôt qu'un «changement apporté en vue du rétablissement d'une forme primitive ou ancienne» (cf. http://atilf. atilf.fr/tlf.htm sub $v$. réforme).

7. B. H. RosenweIn, «Y avait-il un 'moi' au haut Moyen Âge?», Revue historique, 633 (2005), p. 46. 
reconnaît plus tel qu'il était, il ne se sent plus étranger à la communauté, il n'est plus étranger à la communauté. Il en fait partie, intégralement.

\section{Les Dialogues de Grégoire}

La production des normes - et en particulier des normes monastiques dans le discours hagiographique a été étudiée par Bruno Judic à partir des livres II et IV des Dialogues lors du récent colloque Normes et hagiographie ${ }^{8}$, la présentation de la règle de Benoît dans le livre II l'occupant essentiellement. En tant qu'auteur et qu'autorité, Grégoire apporte son poids à la règle. La diffusion franque et anglaise de la règle de Benoît au viI ${ }^{\mathrm{e}}$ siècle doit beaucoup à l'autorité de Grégoire, qui met en scène et installe Benoît en tant que norma rectitudinis (Dialogues II, 3, 2) ${ }^{9}$.

On retrouve volontiers le terme «réforme» sous la plume des auteurs qui évoquent l'action de Grégoire, du point de vue de l'administration des Églises dont les prélats et souverains sont ses correspondants ${ }^{10}$, mais aussi avec un sens moral, voire ecclésial ${ }^{11}$. Les miracles peuvent ainsi associer la pureté sacrale de l'Église, pourvue de ses rituels fondateurs, et celle du chrétien (ou plus encore de la chrétienne), qui doit se garder de l'impureté souvent accolée à la sexualité, comme dans l'histoire de la jeune mariée de Tuscia qui se rend à la dédicace d'une chapelle à l'époque de l'évêque Fortunat de Todi. Comme elle ne s'est pas abstenue d'avoir des rapports conjugaux la nuit précédente, elle est possédée par des démons qui se multiplient à mesure que l'on tente de délivrer la malheureuse par la magie. Arrive Fortunat qui, par ses prières, «la rend aussi saine et sauve

8. B. Judic, «Grégoire le Grand et la production de normes: le cas des Dialogues », dans M.-C. Isaia et T. Granier éd., Normes et hagiographie dans l'Occident médiéval. Colloque de Lyon, octobre 2010, à paraître.

9. Il faut rappeler que cette épithète est accolée à Benoît alors même que les moines qui l'ont choisi comme abbé dans un premier monastère se rebellent face à son autorité et regrettent leurs anciennes mœurs...

10. C. Dagens, Saint Grégoire..., p. 331 («Il est sûr qu'il a été de plus en plus préoccupé par la réforme de l'Église et la conversion des peuples païens »); L. PIETRI, «Grégoire le Grand et la Gaule : le projet pour la réforme de l'Église gauloise», dans Gregorio Magno e il suo tempo, vol. I, Rome, 1991, p. 108-128; EAD., «La réforme de l'Église du Regnum Francorum» dans EAD. éd., Histoire du christianisme, vol. III, Les Églises d'Orient et d'Occident (432-610), Paris, 1998, p. 873-878; Ph. Bernard, «Sanctus Gregorius Papa...», p. 174 («[...] ces contrées dont les rois et les prélats étaient restés sourds à ses appels à la réforme»).

11. C. STRAw, Gregory the Great: Imperfections in Perfection, Berkeley-Los Angeles-Londres, 1991, p. 71 («Gregory has his own particular agenda of spiritual reform. He wishes to strengthen and purify the corporate life of the church by emphasizing the importance of hierarchical order and obedience to authority ). Carole Straw intitule par ailleurs le chapitre X de son livre «Reform and the preacher». 
que si le diable n'avait jamais exercé sur elle de droit de propriété » (Dialogues I, $10,5)$.

La nature hagiographique de l'œuvre, dans laquelle la règle de saint Benoît est omniprésente, et l'importance des miracles jouent par ailleurs un rôle essentiel dans la mise en scène du respect de cette regula. De nombreux miracles montrent Benoît rappelant ses moines à l'ordre, car il a été informé de façon extraordinaire de leurs actes ou même de leurs pensées les plus intimes. La surprise causée chez les moines, qui sont témoins de la scène et qui prennent donc acte de la faute de leur coreligionnaire, renforce l'autorité de Benoît et manifeste la nécessité de se conformer à sa volonté. Les mouvements de dépit des moines qui supportent mal le devoir d'obéissance (car il semble les ravaler au rang d'esclaves) ou qui commettent des étourderies aussitôt oubliées, n'échappent pas à Benoît: il les expose immédiatement (Dialogues II, 19-20). Nous n'insisterons donc pas sur ce point.

On trouve peu dans les Dialogues l'appel à un retour aux temps apostoliques ou à des normes anciennes, malgré la présentation de saints des temps anciens dans le livre III. On ne peut donc parler d'une réforme prônée par Grégoire au sens strict où on l'entend pour les ordres religieux. Il y a bien cependant, chez Grégoire, un passé à partir duquel il souhaite refonder la vie des clercs et des laïcs. Il s'agit de sa propre retraite monastique, dont il a été tiré trois ou quatre ans avant l'écriture des Dialogues pour devenir pape. Et lorsqu'il rédige son œuvre (en 593-594), le grand monastère bénédictin, le Mont-Cassin, a été détruit par la conquête lombarde en 581-589. L'écriture hagiographique rappelle le temps de la règle, dont la rédaction est mise sur le même plan que les miracles, dans la lutte du saint contre le mal ${ }^{12}$.

Le regret que Grégoire a de son ancienne vie monastique le pousse à rechercher les perfections dans les miracles de saints italiens, qui opèrent une conversion personnelle spectaculaire ou contribuent à étendre aux laïcs les préoccupations monastiques. Le premier saint homme évoqué dans les Dialogues est ainsi un abbé fondateur, Honorat de Fondi. Le régime extrême que s'impose Honorat dès son enfance est d'abord un objet de moquerie pour son entourage. Il devient cependant un modèle, par la grâce d'un miracle qui touche précisément la nourriture : un poisson apparaît en pleine montagne et lui permet de s'abstenir

12. T. GranIER, «Saints fondateurs, récits d'origine et légendes apostoliques dans l'Italie méridionale des $\mathrm{VIII}^{\mathrm{e}}-\mathrm{XII}^{\mathrm{e}}$ siècles », dans E. BozOKy éd., Hagiographie, idéologie et politique au Moyen Âge en Occident (Actes du colloque international du Centre d'Études supérieures de Civilisation médiévale de Poitiers. 11-14 septembre 2008), Turnhout, à paraître. Sur les miracles, voir aussi : S. Boesch Gajano, «Demoni, miracoli e Gregorio Magno », dans Hagiographie, culture et sociétés (Colloque de Nanterre de 1979), Paris, 1981, p. 263-281; W. McCREADY, Signs of Sanctity. Miracles in the Thought of Gregory the Great, Toronto, 1989. 
de manger la viande qui avait été préparée. La première question que pose l'interlocuteur de Grégoire, le diacre Pierre, est alors celle-ci: «Devons-nous penser qu'un homme si distingué, avant d'être maître de disciples, eut lui-même un maître?» (Dialogues I, 1, 5). Honorat n'a pas eu de maître, mais Grégoire souligne bien que cela ne doit pas constituer un exemple: il avait reçu un don particulier de l'Esprit. Toute communauté doit être guidée par un maître qui se réfère à une discipline bien établie. Grégoire lui-même, après avoir mené sa communauté du mont Caelius, apporte ses principes de gouvernement aux Églises occidentales.

On ne peut cependant voir dans l'activité de Grégoire une action concertée à l'échelle d'une Église guidée par Rome, c'est bien connu, et cela même si la figure de Grégoire le Grand fut une source d'inspiration pour Grégoire VII. Le terme reformare lui-même n'est guère utilisé dans les Dialogues, mais son usage dans les Moralia correspond bien à ce qui est au cœur du discours hagiographique de Grégoire le Grand: la renaissance, la réforme personnelle, alors que Grégoire VII désigne toujours la réforme de l'Église dans son entier ${ }^{13}$. Cela n'empêche pas que le projet de réforme personnelle et de conversio de Grégoire résonne dans ses tentatives d'administration des Églises d'Occident, que l'on peut appréhender dans les œuvres hagiographiques qui se réfèrent aux Dialogues.

La plupart des thèmes qui ont rapport à la bonne administration de l'Église et à sa purification sont ainsi bien présents dans les Dialogues, même si on les retrouve exposés sous une forme beaucoup plus disciplinaire dans la Règle pastorale et dans le Registre des lettres de Grégoire le Grand. Les rapports entre l'œuvre hagiographique de Grégoire et ses autres travaux sont étroits, malgré les apparences ${ }^{14}$. Si l'on ne peut parler de réforme religieuse au sens propre, la correspondance du pape montre d'ailleurs que Grégoire a eu une volonté constante de promouvoir à l'échelle de l'ensemble de l'Occident une réforme

13. G. B. LADNER, «Gregory the Great and Gregory VII : A comparison of their concepts of renewal», Viator, 4 (1973), p. 1-31; G. ConSTABLE, «Renewal and Reform in religious life. Concepts and realities», dans R. L. BENSON et al. éd., Renaissance and Renewal in the Twelfth Century, Cambridge (MA), 1991, p. 38.

14. Sur la complémentarité et les convergences des Dialogues avec les autres œuvres de Grégoire le Grand, cf. A. DE VoGÜÉ, «Introduction», dans GrÉGOIRE LE Grand, Dialogues, éd. citée, p. 29-31. On retrouve en particulier une convergence de mise en œuvre et de thèmes dans l'usage par Grégoire des exempla: B. Judic, «Grégoire le Grand et la notion d'exemplum», dans M. A. Polo de Beaulieu, P. Collomb et J. Berlioz éd., Le Tonnerre des exemples. Exempla et médiation culturelle dans l'Occident médiéval, Rennes, 2010, p. 131-142. Les Dialogues conservent cependant une grande originalité, notamment dans leur espace géographie, centré sur Rome, qui «confirme ce qui ressort de l'activité politique de Grégoire» (S. Boesch Gajano, Grégoire le Grand. Aux origines du Moyen Âge, Paris, 2007, p. 98). Plus généralement, cf. J. M. Petersen, The Dialogues of Gregory the Great in their Late Antique Cultural Background, Toronto, 1984. 
administrative et morale aux thèmes constants. Grégoire s'adresse donc non seulement aux moines, à qui sont destinés prioritairement les Dialogues, mais aussi aux différents rois, qui étaient capables de faire infléchir le comportement des Églises nationales ${ }^{15}$. Parmi ceux-ci, on peut surtout citer les Mérovingiens de la branche austro-burgonde, le roi des Wisigoths Reccared et le roi du Kent Æthelberht. En la matière, les Dialogues montrent que la figure de l'autorité n'a pas nécessairement besoin d'être catholique. Grégoire le Grand recourt en effet à plusieurs reprises à la figure de l'Ostrogoth Totila pour démontrer que même un roi hérétique peut respecter les saints et protéger les églises. Totila reste un personnage ambivalent dans les Dialogues, car le pape n'entend pas donner un trop bon rôle à un personnage arien. Pour autant, l'Ostrogoth ne constitue pas un repoussoir absolu: un roi, même mauvais, reste un instrument de la volonté de Dieu ${ }^{16}$. Grégoire le Grand choisit aussi d'adresser son message aux grands aristocrates, qui constituaient à la fois les auxiliaires des souverains et les partenaires habituels des églises locales. Même loin de l'Italie, Grégoire le Grand sut entrer en relation avec de hauts personnages comme le patrice de Provence Dynamius ou le duc wisigoth Claudius ${ }^{17}$.

Si l'influence de Grégoire en Occident est souvent mesurée à l'aune de sa correspondance et de ses réflexions théologiques et exégétiques, de sa pastorale, on ne saurait sous-estimer le rôle des Dialogues. Martin Heinzelmann a bien rappelé la diffusion importante des collections de «Vies de Pères » (Vitae Patrum), et en particulier des Dialogues, dans la Gaule du haut Moyen Âge. Même de ce côté des Alpes, les Dialogues occupent une place tout à fait essentielle au sein des manuscrits hagiographiques conservés des VII ${ }^{\mathrm{e}}$ et VIII ${ }^{\mathrm{e}}$ siècles ${ }^{18}$. Le statut de ce texte dans son rapport à la sainteté et aux reliques doit aussi être souligné. À Saint-Wandrille, on mentionne déjà, dans le troisième quart du viI ${ }^{\mathrm{e}}$ siècle, des reliques que l'on a fait venir de Rome en même temps que des livres sur les saints (volumina diversa sanctarum Scripturarum) du pape Grégoire le Grand ${ }^{19}$.

15. Sur le rôle des souverains dans la conversion et la christianisation, cf. B. DumÉżL, Les Racines chrétiennes de l'Europe. Conversion et liberté dans les royaumes barbares (V $V^{e}$-VIII siècle), Paris, 2005, p. 171-358 («Le retour du roi»).

16. Par exemple: Dialogues III, 5-6, où le roi Totila alterne cruauté puis aménité envers les saints.

17. GrÉGoIre le Grand, Ep. III, 33 (à Dynamius); ibid., IX, 230 (à Claudius).

18. M. Heinzelmann, «L'hagiographie mérovingienne. Panorama des documents potentiels», dans M. Goullet, M. Heinzelmann et C. Veyrard-Cosme éd., L'Hagiographie mérovingienne à travers ses réécritures, Ostfildern, 2010 p. 27-82 (p. 27-31).

19. Chronique des abbés de Fontenelle I. 6 ; cf. M. Heinzelmann, «Pouvoir et idéologie dans l'hagiographie mérovingienne», dans E. BozoKy éd., Hagiographie, idéologie et politique..., à paraître. 
Les Dialogues ne sont pas, en eux-mêmes, une œuvre qu'on pourrait qualifier d'emblée de réformatrice. Il n'empêche qu'ils sont porteurs de thèmes que l'on sait chers à Grégoire le Grand: la lutte contre la simonie, ou, pour être exact, contre un usage inadéquat de l'argent par les clercs; la lutte contre le nicolaïsme sous toutes ses formes, et notamment celui qui se manifeste par le contact trop proche entre clercs et moniales; la volonté d'exclure les laïcs de la gestion de l'Église et notamment de ses propriétés; un effort de hiérarchisation de l'Église ou, au moins, de respect étroit de la hiérarchie en place; enfin, une volonté de moralisation de la société, ce dernier thème étant illustré par une utilisation didactique des visions eschatologiques, surtout dans le livre IV des Dialogues.

Si on ne peut, bien évidemment, parler de centralisation de l'Église au $\mathrm{VII}^{\mathrm{e}}$ siècle, on doit souligner le soin avec lequel Grégoire le Grand mena son action sur tout le terrain occidental par l'envoi de légats (en Gaule et en Espagne), dont la figure la plus éminente est sans doute l'abbé Cyriaque ${ }^{20}$. Le pape utilise également ses propres œuvres, qu'il diffuse largement. La Regula Pastoralis arriva rapidement en Gaule du Sud, portée par un messager de Rome, et les Moralia in Job furent adressées à Léandre de Séville. Rien ne prouve que les Dialogues aient suivi le même chemin, mais on les retrouve assez précocement là où le pape avait prodigué ses conseils. Au viI ${ }^{\mathrm{e}}$ siècle, l'œuvre est en effet connue aussi bien en Espagne wisigothique, en Gaule que dans le monde anglosaxon. Sans reprendre les analyses poussées de Bruno Judic sur la question de la postérité grégorienne ${ }^{21}$, on peut se contenter d'examiner trois œuvres, provenant de trois espaces différents, afin de voir comment les thèmes romains ont pu être pérennisés ou transformés par leur milieu de réception. Il s'agit ainsi de voir si le genre hagiographique, a priori hautement conservateur, a permis la diffusion du message à connotation réformatrice.

20. C. et L. Pietri, Prosopographie de l'Italie chrétienne (313-604), Rome, 1999, p. 523-525.

21. B. Judic, «Totius Europae Speculator. Habilitation à diriger des recherches. Présentation par l'auteur», Revue du Nord, 81 (1999), p. 606-613; ID., «La tradition de Grégoire le Grand dans l'idéologie politique carolingienne», dans R. LE JAN éd., La Royauté et les élites dans l'Europe carolingienne (du début du IX'aux environs de 920), Villeneuve d'Ascq, 1996 (p. 31-35 à propos de reprises du livre IV des Dialogues dans un florilège attribué à Haymon); ID., «Grégoire le Grand, Alcuin et l'idéologie carolingienne», dans W. FALKOwski et Y. SASSIER éd., Le Monde carolingien: Bilan, perspectives, champs de recherches (Actes du colloque de Poitiers, 18-20 novembre 2004), Turnhout, 2009, p. 105-120; ID., «Grégoire le Grand, Alcuin, Raban et le surnom de Maur», dans P. Depreux, S. LebecQ, M. Perrin et O. Szerwiniack éd., Raban Maur et son temps, Turnhout, 2010, p. 31-48. 


\section{Les Vies des Pères de Mérida}

Pour ce qui concerne la postérité hagiographique immédiate des Dialogues, commençons par l'œuvre la plus ancienne, à savoir les Vies des Pères de Mérida, écrites par un diacre anonyme de l'école ecclésiastique de Sainte-Eulalie de Mérida ${ }^{22}$. Dans sa préface, son auteur se réclame explicitement des Dialogues et prétend avoir pris l'œuvre de Grégoire le Grand pour modèle ${ }^{23}$. Cet auteur espagnol prétend écrire sous l'épiscopat du troisième successeur de Masona de Mérida, c'est-à-dire sans doute dans la quatrième décennie du VII ${ }^{\mathrm{e}}$ siècle, vers 633-638 ${ }^{24}$.

L'œuvre est a priori fort grégorienne. L'auteur affirme en effet vouloir écrire un équivalent espagnol aux Dialogues, de façon à montrer à ses lecteurs que les prodiges ne se sont pas seulement déroulés aux temps anciens ou dans la lointaine Italie. Malheureusement, le diacre de Mérida ne parvient pas à respecter longtemps son projet. Il ne respecte pas la forme dialoguée, ni ne tient la structure de l'ouvrage. Après avoir recueilli trois miracles d'origines variées, il se met à raconter la vie des évêques de Mérida et entre dans un genre littéraire proche des Gesta avec fort peu d'incursions du surnaturel.

Chez le diacre espagnol, la fidélité aux principes réformateurs est tout aussi relative que son respect de la forme. Certains thèmes grégoriens sont pourtant bien présents. Par exemple, le premier récit des Vies des Pères de Mérida rapporte une vision eschatologique détaillée, avec visite de l'au-delà et description de la récompense des justes et de la punition des méchants ${ }^{25}$. L'esprit de ce récit est très proche du livre IV des Dialogues de Grégoire. De même, le second miracle de Mérida concerne la punition d'un moine coupable de gula, ce qui renvoie là encore au modèle grégorien (Dialogues II, 12). Le troisième miracle raconte comment le saint abbé Nanctus avait fait le vœu de ne jamais regarder une femme: le passage semble inspiré de Dialogues II, 3, qui évoque un même vœu fait par Martin de Campanie ${ }^{26}$.

22. Le recueil donne les biographies des évêques de Mérida depuis le milieu du vi ${ }^{\mathrm{e}}$ siècle: Vitas sanctorum patrum Emeretensium, éd. A. MAYA SÁnchez, Turnhout, 1992; cf. P. HENRIET, «Écrire l'histoire des évêques en Péninsule ibérique, de l'époque wisigothique à la 'normalisation de l'Église' (VII-XII ${ }^{\mathrm{e}}$ siècle)», dans F. BougARD et M. Sot éd., Liber, gesta, histoire. Écrire l'histoire des évêques et des papes, de l'Antiquité au XXI siècle, Turnhout, 2009, p. 329-346.

23. Vitas sanctorum patrum Emeretensium, p. 3-4.

24. Thomas Deswarte souligne que, malgré cela, il faut se garder de considérer que le culte de Grégoire ait été développé de façon particulièrement précoce dans les chrétientés hispaniques (Une Chrétienté romaine sans pape. L'Espagne et Rome (586-1085), Paris, 2010, p. 98). Sur la présence des différentes œuvres de Grégoire en Espagne au haut Moyen Âge, cf. ibid., p. 103-104.

25. Vitas sanctorum patrum Emeretensium, 1.

26. On sait aussi que saint Benoît subit la pire tentation de sa vie lorsqu'il se souvient d'avoir vu une femme (Dialogues II, 2). 
La lutte contre le nicolaïsme sous toutes ses formes est assurément une thématique majeure des Vies des Pères de Mérida. Le rédacteur sait toutefois que ce sujet est devenu particulièrement brûlant et peut dériver vers l'hérésie encratique; le précédent priscillianiste l'inquiète peut-être aussi. Il se voit donc obligé de préciser que les saints espagnols ne méprisent pas les femmes en général, mais redoutent seulement la tentation.

Pour autant, on voit le clergé de Mérida soupçonner sans cesse son évêque de trop fréquenter les femmes, sans doute dans l'espoir d'obtenir la déposition du coupable. Pour désamorcer la crise engendrée par ce raidissement intéressé de la morale sexuelle, les Vies des Pères de Mérida rapportent un cas limite, celui de l'évêque de Mérida, Paul. Dans les années 570, cet ancien médecin oriental était connu pour ses talents d'obstétricien. Après son ordination, une femme supplia Paul de venir la délivrer d'un enfant mort-né en pratiquant une césarienne. Paul pria longtemps Dieu de l'éclairer avant d'accepter de faire ce geste médical qui lui imposait de voir une femme nue ${ }^{27}$. On comprend là le raisonnement: même si la lutte contre le nicolaïsme était louable en soit, il restait possible pour un clerc de briser l'interdit si une vie humaine en dépendait. Voilà qui permettait de relativiser - et même de contredire - le passage des Dialogues où Grégoire le Grand avait vanté le comportement de moines italiens qui avaient renoncé à entrer dans la clôture d'un monastère féminin pour guérir une moniale malade (Dialogues I, 4, 4).

Sur la question de la simonie, l'auteur des Vies des Pères de Mérida est beaucoup plus gêné. Il sait que le phénomène existe, que plusieurs évêques en ont profité, mais aussi que Grégoire réprouve le procédé. Pour autant, il n'ose pas remettre en cause la sainteté déjà bien établie des prélats espagnols. Dès lors, il cherche à justifier des pratiques bien peu canoniques. Par exemple, l'évêque Paul voulait que son neveu Fidelis lui succède. Pour en être sûr, Paul fit un testament accordant un legs important au clergé de Mérida si celui-ci donnait son aval à la nomination de Fidelis ; si les clercs préféraient revenir à une libre élection, cela restait possible, mais ils n'auraient alors aucun sou de l'héritage. L'auteur en est réduit à expliquer que le dispositif n'était qu'une ruse, qui prenait en compte la cupidité bien connue des clercs locaux et visait à faire élire un homme qui n'était pas touché par ce péché ${ }^{28}$.

Les évêques espagnols manient pourtant l'argent et pratiquent même l'usure. Une fois encore, cela gêne notre hagiographe, puisque chez Grégoire, les saints italiens étaient pauvres et les usuriers du côté du mal. Vaille que vaille, l'auteur des Vies des Pères de Mérida tente de montrer les évêques espagnols sous un meilleur jour encore: ainsi, à la fin de sa vie, l'évêque Masona accepta

27. Vitas sanctorum patrum Emeretensium, 4,2 .

28. Ibid., 4,4 . 
de rendre leurs reconnaissances de dettes à toutes les personnes qui lui devaient quelque chose. En termes financiers, le geste était encore plus beau que celui de Benoît.

Le rapport entre le saint homme et la figure de l'autorité civile pose moins de difficultés à l'auteur des Vies des Pères de Mérida, adoptant l'idée que tout roi est institué par Dieu. Prenant modèle sur le Totila de Grégoire le Grand, les Vies des Pères de Mérida dressent ainsi un portrait tout en nuances du roi arien wisigoth Léovigild ${ }^{29}$ : le souverain apparait assurément cruel dans ses contacts avec Masona, mais il reconnaît les pouvoirs miraculeux des saints chrétiens comme Nanctus, et il accorde des donations aux églises... Léovigild y est un grand roi, même s'il s'agit d'un mauvais roi ${ }^{30}$.

Le choix des personnages évoqués par les Vies de Pères de Mérida reste toutefois, dans l'ensemble, fidèle aux conceptions de Grégoire. On y trouve une juste répartition entre les miracles accomplis par les moines et par les évêques, tandis que les adjuvants des saints sont tantôt les rois, tantôt les grands laïcs. Parmi ceux-ci, les Vies de Pères de Mérida prennent soin de faire figurer le duc Claudius ${ }^{31}$, un personnage qui avait certes été le grand général de Reccared, mais également le correspondant laïque de Grégoire le Grand en Espagne.

En somme, le modèle hagiographique grégorien n'est donc pas véritablement trahi par sa transposition wisigothique. La plupart des idées réformatrices sont maintenues, même si certaines sont quelque peu tempérées pour s'adapter aux réalités locales. Le projet de l'hagiographe de Mérida, qui était de proposer une transposition régionale de l'hagiographie romaine, est finalement réalisé avec un certain succès.

\section{La Passio de Praejectus}

En Auvergne, peu après 680, l'auteur de la Passion de saint Praejectus de Clermont affirme également avoir lu les Dialogues de Grégoire le Grand et prétend s'en inspirer ${ }^{32}$. En elle-même, cette revendication est probablement

29. Ibid., $3 ; 4,4-5$ et 9 .

30. En ce qui concerne les rapports des saints avec l'exercice du pouvoir et les souverains et leur image biaisée dans les sources hagiographiques hispaniques de l'époque, cf. P. HENRIET, «Un horizon hagiographique d'opposition au pouvoir. Les milieux monastiques et ascétiques de l'Espagne septentrionale au VII siècle», dans E. Bozoky éd., Hagiographie, idéologie et politique..., à paraître.

31. Vitas sanctorum patrum Emeretensium, 4, 10-11.

32. Passio Praeiecti (BHL 6915-16), éd. B. Krusch, Hanovre-Leipzig, 1910 (MGH SS rer. Merov., V), p. 212-248; cf. P. FouraCre et R. A. GERBERding éd., Late Merovingian France. History and Hagiography, 640-720, Manchester, 1996, p. 254-270 pour le commentaire de la Passion et p. 271-300 pour la traduction anglaise du texte. Une traduction française du texte sera donnée dans le volume que prépare l'équipe d'HagHis sur le dossier de saint Léger. 
correcte, dans la mesure où il emprunte sa liste d'auctoritates à la préface à la Vie de Colomban de Jonas de Bobbio, à laquelle il ajoute l'œuvre de Grégoire le Grand qu'il qualifie de récente. Bref, pour un écrivain de la fin du VII siècle, les Dialogues apparaissent comme le nouveau modèle hagiographique.

Sur l'auteur de la Passion de saint Praejectus, nous ne savons que peu de choses. Il semble évoluer dans un milieu monastique local, influencé très indirectement par le courant irlandais. Ses lectures sont à la fois vastes et désordonnées. Avec des moyens littéraires que l'on devine assez limités, il a reçu la mission d'écrire une œuvre qui permette à la société de Clermont de se réconcilier autour d'une figure de sainteté fédératrice. Notre hagiographe se trouve donc obligé de dire du bien de Praejectus, mais aussi des aristocrates commanditaires du meurtre de Praejectus (qui se sont excusés depuis et qu'il faut donc féliciter). L'hagiographe doit aussi mettre en valeur les disciples de Praejectus, qui adhèrent au mouvement colombanien, mais aussi la figure du successeur de l'évêque, Avitus, qui appartient au parti opposé. Pire encore, notre auteur doit célébrer la mémoire de Léger d'Autun, que Praejectus a pourtant contribué à faire condamner à mort.

La complexité de cette tâche conduit à une œuvre maladroite. Pour autant, certains thèmes grégoriens sont bien présents. On apprend ainsi qu'aux temps où il était prêtre du diocesis d'Issoire, Praejectus de Clermont se retirait souvent dans un monastère où «il évitait la vue des laïcs» (Passio Praeiecti, 5). Du point de vue de la pastorale, une telle attitude était probablement désastreuse, si tant est qu'elle fût authentique. Mais, apparemment, notre hagiographe a tenu à illustrer le thème de la séparation totale entre clerc et laïc, sans bien percevoir que le topos convenait plutôt au traitement d'un saint suivant une règle monastique. Sur le rapport à l'argent, l'hagiographe s'en sort un peu mieux. Comme le saint Benoît des Dialogues, Praejectus multiplie les pièces de monnaie quand le besoin s'en fait sentir. Et, comme Benoît, Praejectus prend la direction d'un petit monastère ${ }^{33}$.

La question de la libre élection épiscopale tarabuste beaucoup plus l'hagiographe auvergnat. Celui-ci connaît les principes canoniques édictés par le concile de Nicée. Praejectus, parce qu'il est saint, doit donc avoir une élection canonique, sans recours à la simonie et surtout sans intervention d'un pouvoir laïc. L'hagiographe se sent donc obligé de raconter par le menu l'accès de son héros au pontificat. On apprend ainsi qu'à la mort de l'évêque Félix, le candidat naturel au siège de Clermont est l'archidiacre Garivald. Celui-ci a même anticipé son élection en demandant aux cinq clercs les plus éminents de Clermont de souscrire un document lui accordant leur suffrage. Or, Praejectus a lui-même mis son nom sur cet accord, ce qui entre dans le champ des conjurationes interdites 
par les canons mérovingiens. Prenant soudainement conscience de son erreur, Praejectus se met à raconter partout que sa mère a eu une vision montrant qu'il était promis à l'épiscopat. Il parvient ainsi à convaincre les clercs du collège électoral de lui donner leurs voix. Garivald, se sentant pris de court, doit alors acheter à prix d'or le suffrage des aristocrates qui composent la partie laïque du collège électoral. Il gagne ainsi l'élection et réussit à être ordonné, même s'il meurt quarante jours plus tard. Châtiment divin à l'encontre d'un pécheur? Non, dit l'hagiographe qui ne veut décidément blesser personne, puisque Garivald était un homme «de sainte mémoire». Dieu voulait simplement que Praejectus dispose d'une élection canonique. Malheureusement, pour remplacer le défunt Garivald, le Palais se mêle maintenant de l'affaire auvergnate et ordonne que le comte de la ville soit nommé évêque ${ }^{34}$. Un simple laïc accédant à l'épiscopat, c'était là le type de situation que Grégoire le Grand reprochait le plus au monde franc $^{35}$. Par chance, le comte «fut pris de crainte à l'idée d'agir contre les décrets des canons et fit une déclaration publique dans laquelle il s'affirmait indigne de l'épiscopat ${ }^{36}$. Voilà un grand laïc qui accepte les principes réformateurs ou, peut-être, qui ne veut pas d'un poste qu'il devine piégé. À l'issue d'un nouveau débat au sein du collège électoral, et sans doute faute de candidats, on se tourne enfin vers Praejectus. La morale est sauve: l'élection a bien été canonique ${ }^{37}$.

Débuté sous de tels auspices, l'épiscopat de Praejectus ne peut que mal se dérouler. L'évêque est contesté au niveau local en raison de son origine sociale peu élevée, et malmené au niveau national en raison aussi de son alliance avec la reine Chimnéchilde, patronage douteux que l'auteur avait eu tout de même la prudence de dissimuler au moment de l'élection ${ }^{38}$. L'hagiographe s'en trouve réduit à ne pas trop raconter l'épiscopat et à se hâter vers la passion. Un bon martyre permettrait en effet de réunir la tradition colombanienne et le vieux fond du passionnaire gaulois. Malheureusement, l'affaire qui provoque la chute de Praejectus touche à la question sensible des biens d'Église. Par sa prédication, Praejectus avait en effet attiré à lui une aristocrate nommé Claudia; l'évêque lui avait donné la bénédiction des veuves et gérait une partie de sa fortune. Mais le patrice de Provence, Hector, guignait lui aussi sur l'héritage; il enleva la fille de

\section{Passio Praejecti, 13-14.}

35. Grégoire le Grand, Ep. VIII, 4 à la reine Brunehaut, septembre 597 (CC 140A, p. 520): «Sed et hoc uobis curae sit ut, quia nostis, neophytum egregius praedicator ad sacerdotii regimen omnino uetat accedere, nullum ex laico patiamini episcopum consecrari.»

36. Passio Praejecti, 14.

37. Premières réflexions sur cette description de l'élection de Praejectus: E. VACANDARD, «Les élections épiscopales sous les Mérovingiens », Revue des Questions Historiques, $32^{\mathrm{e}}$ année, 19 (1898), p. 373-376.

38. Ce patronage n'est évoqué que lors du procès qui oppose Praejectus à Hector de Marseille (Passio Praejecti, 24). 
Claudia, l'épousa et revendiqua la fortune. Praejectus l'accusa de rapt, Hector se rebiffa en l'accusant d'avoir usurpé l'héritage de Claudia ${ }^{39}$.

Selon le droit romain, Hector avait sans doute raison : Claudia ne pouvait pas totalement déshériter sa fille au profit de l'évêque. La Lex Falcidia, passée dans le Code Théodosien, interdit à un testateur d'écarter sa descendance de l'héritage ${ }^{40}$. Mais, ici, la logique réformatrice est à l'œuvre: pour Praejectus, les biens d'une femme consacrée à Dieu appartiennent déjà à Dieu. En cela, la pratique de Praejectus peut rappeler de nombreuses affaires d'héritages et de donations italiennes qu'a traitées Grégoire en faveur de son Église. Grégoire connaissait en effet très bien le droit des héritages mais ne le respectait pas à la lettre au sujet des dons faits (par de saintes femmes en général) à l'Église, considérant qu'il s'agissait de cas particuliers ${ }^{41}$. Le pape, entouré dans sa jeunesse par sa mère et ses tantes, très présentes, s'est par ailleurs particulièrement intéressé aux moniales ${ }^{42}$. Un simple laïc ne saurait bénéficier de terres qui semblaient promises à l'Église, d'autant plus s'il s'engage pour cela dans un mariage illégal qui peut être taxé de rapt, comme c'est le cas d'Hector dans la Passio. Une longue affaire judiciaire s'en suit, qui est le passage le plus connu de la vie de Praejectus. L'évêque de Clermont parvient à faire condamner Hector, mais il déclenche se faisant une suite de violences dont saint Léger d'Autun est la victime collatérale. Praejectus finit lui-même par être assassiné par un groupe d'aristocrates proches de Léger.

Son successeur à Clermont, Avitus, qui appartenait au parti victorieux, se chargea de normaliser la situation et de régulariser ce qui pouvait l'être. Il rappela d'exil les moines compagnons de Praejectus, fit construire pour eux un monastère et leur imposa une Règle ${ }^{43}$. Le temps du monachisme gyrovague était révolu: dans un esprit très chalcédonien, l'autorité épiscopale entendait encadrer les pratiques monastiques. La Passio entérine à sa façon l'échec du courant irlandais que représentait Praejectus.

En fin de compte, la Passio Praejecti correspond à une nouvelle forme de régionalisation de l'hagiographie romaine. Les Dialogues y apparaissent comme un modèle littéraire beaucoup plus lointain que dans le cas des Vies des Pères de Mérida; mais, a contrario, les thématiques réformatrices sont beaucoup plus abouties qu'en Espagne. Toutefois, sans doute ne faut-il pas voir là la seule influence de la pensée de Grégoire le Grand. Depuis le vie siècle, l'Église

39. Passio Praejecti, 23.

40. Selon la Lex Falcidia, un quart de l'héritage devait revenir aux descendants: C. Th. II, 19, 4; V, 1, 4; IX, 14, 3; XVI, 8, 28.

41. J. Richards, Consul of God. The Life and Times of Gregory the Great, Londres, 1980, p. 110.

42. J. Martyn, Pope Gregory and the Brides of Christ, Cambridge, 2009 (part. p. XI).

43. Passio Praejecti, 34. 
gauloise cherche en effet à se réformer par l'entremise des conciles nationaux, mais selon des thèmes communs à ceux de Grégoire le Grand. En somme, il est bien difficile d'apprécier si l'effort réformateur de l'hagiographe auvergnat constitue le produit de Rome, des conciles d'Orléans et Paris ou d'une volonté générale de retour aux temps de Nicée et Chalcédoine.

\section{La première Vie de Grégoire le Grand}

Quittons maintenant la Gaule pour aborder un troisième espace où les Dialogues de Grégoire le Grand connaissent une excellente diffusion: l'Angleterre anglo-saxonne, que le pape a travaillé à convertir ${ }^{44}$. Les Dialogues y sont connus au plus tard dès le début du viII ${ }^{\mathrm{e}}$ siècle; signe de leur succès, le roi Alfred les fait traduire en vernaculaire vers 891. C'est dans cet espace qu'un moine du prestigieux monastère de Whitby compose la première Vie de Grégoire le Grand, sans doute entre 704 et $714^{45}$.

Le manque d'information de cet auteur sur le personnage historique de Grégoire est criant et, dans l'ensemble, il ne cherche pas à le dissimuler. Son œuvre est moins le récit d'une vie, dans la stricte tradition de l'hagiographie épiscopale, qu'un assemblage d'anecdotes appelant de la part de l'auteur des réflexions morales ou eschatologiques. En raison de cette forme très particulière, l'œuvre se trouve naturellement amenée à puiser son inspiration dans les Dialogues. Même si elle ne va pas jusqu'à reprendre la forme dialoguée, l'écriture de l'hagiographe est très fidèle au style grégorien. Au chapitre 23 , le moine expose par exemple qu'il ne cite pas les paroles des personnages de façon littérale, mais qu'il en donne le sens général, de façon à ce que la portée spirituelle de l'énoncé soit plus facile à percevoir. Il s'agit là d'une reprise délibérée de la Préface des Dialogues.

Outre la forme, le contenu de la Vie du moine de Whitby présente des thématiques assurément grégoriennes. Doit-on parler de conceptions

44. N. Chadwick, «Gregory the Great and the Mission to the Anglo-Saxons», dans Gregorio Magno e il su tempo, t. I, Rome, 1991, p. 199-212; R. MARкus, «Gregory the Great and a Papal Missionary Strategy», dans G. J. Cuming éd., The Mission of the Church and the Propagation of the Faith, Cambridge, 1970 p. 29-38; ID., «The Chronology of the Gregorian Mission to England: Bede's Narrative and Gregory's Correspondence», Journal of Ecclesiastical History, 14 (1963), p. 16-30 [rééd. dans ID., From Augustine to Gregory the Great. History of Christianity in the Late Antiquity, Londres, 1983]; I. N. Wood, «The Mission of Augustine of Canterbury to the English », Speculum, 69 (1994), p. 1-17.

45. Vita Gregorii (BHL 3637), éd. et trad. B. Colgrave, The Earliest Life of Gregory the Great by an Anonymous Monk of Whitby, Lawrence, 1968. Cf. M. LAPIDGE et R. C. Love, «The Latin Hagiography of England and Wales (600-1550)», dans G. PhILIPPART éd., Hagiographies III, Turnhout, 2003, p. 212; et A. THACKER, «Memorializing Gregory the Great: the origin and transmission of a papal cult in the seventh and early eighth centuries », Early Medieval Europe, 7/1 (1998), p. 59-84 (qui suppose que la Vita aurait pour sources des écrits de disciples de Grégoire à Rome). 
réformatrices? Disons au moins qu'il s'agit en tout cas d'idées formatrices pour la jeune Église de Northumbrie. Dès les chapitres 3 et 4, le moine de Whitby se trouve obligé d'annoncer qu'il ne va pas raconter beaucoup de miracles. Il a conscience que ce manque de signes risque de nuire au développement du culte de Grégoire ou, plus prosaïquement, à l'intérêt du lecteur. Il argumente immédiatement en expliquant que ce n'est pas la démonstration surnaturelle qui fait la grandeur du saint; ce faisant, il reprend explicitement un passage des Dialogues, où Grégoire le Grand avait exposé que les miracles accomplis ne témoignent pas de la valeur de ceux qui les font (Dialogues I, 12, 5). La Vie revient à nouveau à cet argument au chapitre 7 , en concluant que la vertu est plus grande que les miracles. Nous sommes ici au cœur de la pensée de Grégoire: le surnaturel permet de réchauffer la foi des chrétiens timides ou d'éveiller celle des infidèles, mais il est inutile pour l'esprit véritablement dévot. Peut-être le moine de Whitby a-t-il aussi à l'esprit la lettre de Grégoire le Grand à Augustin de Cantorbéry, dans laquelle le pape invitait son correspondant à ne pas trop se glorifier de ses miracles ${ }^{46}$.

La Vie du moine de Whitby continue donc son propos en exposant que le véritable exploit accompli par Grégoire le Grand est la conversion du peuple anglais: en effet, la guérison d'une âme constitue une merveille beaucoup plus admirable que la guérison d'un corps. Implicitement, il s'agit d'une référence claire aux Dialogues (III, 17, 7). Dans ce passage, le diacre Pierre venait de s'extasier devant la résurrection d'un mort accomplie par un saint homme italien, mais Grégoire l'avait immédiatement repris en lui exposant que le plus grand miracle, c'est la conversion d'un pécheur. Dans les Dialogues, Grégoire enchaînait en écrivant que le grand miracle du Christ n'était pas la résurrection de Lazare, mais la conversion de saint Paul. Celui qui était un persécuteur était ainsi devenu un modèle de vertu et, surtout, un excellent prédicateur: Paul a en effet réussi à contempler les chœurs angéliques et, en même temps, à proposer une discipline de vie aux simples laïcs. En somme, le vrai miracle, c'est l'apparition d'un bon pasteur. Et c'est là aussi la conception qui sous-tend la réflexion de l'auteur de la première Vie de Grégoire le Grand.

Le moine de Whitby considère qu'un seul type de pouvoir surnaturel est véritablement utile, le don de prophétie (Vita Gregorii, 8). Grégoire le Grand exposait des idées comparables dans le livre II (11) des Dialogues, où il montrait que saint Benoît était devenu véritablement grand à partir du moment où il avait reçu le pouvoir de prévoir les attaques du Diable contre sa communauté. Immédiatement, le moine de Whitby donne une illustration du propos: alors qu'il était encore moine, Grégoire voulut partir convertir des peuples étrangers.

46. Grégoire le Grand, Ep. XI, 36 (CC 140A, p. 927): «Et quicquid de faciendis signis acceperis uel accepisti, haec non tibi sed illis deputes donata, pro quorum tibi salute collata sunt.» 
Mais Dieu lui envoya une sauterelle et Grégoire comprit le message que portait le nom de l'insecte: locusta, soit locus sta, «reste en ce lieu ${ }^{47}$.

Dans l'ensemble, la Vie de Grégoire le Grand entend montrer comment doit se comporter un bon évêque, la tête dans le ciel, les pieds sur terre et l'œil aux aguets. Mais il s'agit aussi de montrer qu'un simple laïc, pour peu qu'il soit vertueux, peut gagner le salut dans la gloire. Grégoire le Grand avait montré dans ses Dialogues que de simples paysans italiens avaient pu mériter le martyre pour être restés fermes dans leur foi face aux Lombards ${ }^{48}$. Le moine de Whitby entreprend pour sa part de démontrer la sainteté du roi Edwin de Northumbrie, qui a porté un haut témoignage de sa foi en se faisant tuer par les païens. Et à la mort d'Edwin, on avait vu son âme s'élever dans le Ciel sous la forme d'un oiseau blanc. L'image est encore une fois empruntée aux Dialogues, qui décrivent des signes comparables lors de la mort de sainte Scolastique (II, 34) et du prêtre Spes (IV, 11).

Au demeurant, Grégoire le Grand avait insisté sur le fait que seul le baptême permet de gagner le salut. Le moine de Whitby ne manque pas, lui aussi, de le rappeler: les seuls revenants qui apparaissent dans des habits glorieux sur le site du champ de bataille de Hatfield sont les âmes de soldats chrétiens (Vita Gregorii, 19).

Certes, il ne suffit pas seulement d'être chrétien pour obtenir le salut. Il faut aussi respecter les normes établies par l'Église. Le chapitre 22 de la Vie de Grégoire raconte la vie d'un laïc romain «qui était riche en biens, mais pauvre en religion »; l'expression elle-même est empruntée aux Dialogues (IV, 40). Ayant voulu divorcer, à l'encontre des règles fixées par l'Église, ce mauvais riche finit excommunié par le pape. Sa tentative de vengeance par l'entremise de magicien échoue piteusement. Les normes matrimoniales sont sévères (même si cette sévérité ne s'applique encore que fort partiellement).

La fin de la Vie de Whitby s'attache essentiellement à commenter la production littéraire de Grégoire le Grand. Il s'agit là de montrer que Grégoire a mis en application dans ses œuvres les qualités qu'il définissait pour être un bon pasteur. Le moine de Whitby voit ainsi dans Grégoire un maître, dont l'enseignement se diffracte dans les différents genres littéraires qu'il a explorés.

47. Le passage a fait l'objet d'un excellent article de B. Judic, «Le corbeau et la sauterelle. L'application des instructions de Grégoire le Grand pour la transformation des temples païens en églises», dans L. MARY et M. Sot éd., Impies et païens entre Antiquité et Moyen Âge, Paris, 2002, p. $97-125$.

48. Grégoire le Grand, Dialogues, III, 28 (SC 260, p. 375); Walter Pohl («Deliberate Ambiguity: The Lombard and Christianity», dans G Armstrong et I N. Wood éd., Christianizing Peoples and Converting Individuals, Turnhout, 2000, p. 50-51) met cependant en doute l'historicité de l'épisode. 
Les différentes réutilisations des Dialogues dans le siècle qui suivit la mort de Grégoire le Grand témoignent de formes d'écritures différentes.

Le moine anglais, quoique étant le plus éloigné dans le temps, est celui qui est le plus fidèle à l'esprit des Dialogues dans toute leur complexité. Il a notamment perçu la portée didactique de l'œuvre, dans laquelle, selon son degré d'approfondissement dans la foi, le lecteur peut aller chercher du surnaturel un peu clinquant (comme le fait le diacre Pierre) ou, au contraire, constater combien l'intervention de Dieu sait se montrer silencieuse, notamment lorsqu'elle se déroule par l'entremise de saints hommes inspirés. Les Dialogues sont une œuvre ouverte et cette dimension, rare dans l'hagiographie, est respectée par la tradition anglo-saxonne. La fonction de son récit est principalement mémorielle et spirituelle. Elle appelle vaguement à la conversion, mais son propos n'a pas de portée véritablement disciplinaire, ni dans l'Église, ni dans le monastère.

Pour les Vies des Pères de Mérida, les Dialogues constituent un modèle aussi indispensable que problématique. L'œuvre est assurément populaire en Espagne, ne serait-ce que parce que le pape a œuvré à la reconstitution de l'Église catholique après la disparition de l'arianisme. Les Vies des Pères de Mérida se trouvent en revanche obligées de louvoyer avec la perspective réformatrice: autant l'eschatologie grégorienne est grandement appréciée, autant sa lutte contre la simonie inquiète un peu. Même la lutte contre le nicolaïsme se voit tempérée d'un appel au calme. Peut-être faudrait-il ajouter que les Dialogues sont sans doute trop monastiques pour l'épiscopat espagnol ; l'auteur des Vies de Mérida se trouva ainsi constamment obligé de faire un tri entre ce qui pouvait être transposé et ce qui ne le pouvait pas.

À côté de ces deux œuvres exceptionnelles, la Vie de Praejectus de Clermont donne peut-être une image de la véritable portée des Dialogues en Occident. L'œuvre de Grégoire le Grand a connu une popularité rapide, au point qu'elle s'impose presque partout comme un modèle. Ce n'est pas pour rien que Grégoire le Grand est connu du monde grec sous le nom de Dialogos. Mais ce large succès ne signifie pas pour autant que les Dialogues aient été compris ou même lus attentivement. Ainsi, la Vie de Praejectus de Clermont est assurément une œuvre réformatrice, mais cette réforme n'est pas vraiment celle que proposait Grégoire le Grand. Le saint gaulois gravite dans un monde monastique, mais son modèle de référence est en Irlande, et non à Rome. Quant à la lutte contre la simonie et l'investiture laïque, elle participe d'un esprit général de réforme, dont Grégoire a en son temps contrôlé le discours, mais qui se retrouve au vII ${ }^{\mathrm{e}}$ siècle dans de multiples bouches. 
Bruno DuMÉzIL - Université Paris-Ouest Nanterre La Défense/IUF (ArScAN - UMR7041), Département d'Histoire, 200 avenue de la République, F-92001 Nanterre

Sylvie Joye - Université de Reims Champagne-Ardenne (CERHIC-EA 2616), Département d'Histoire, 57 Rue Pierre Taittinger, F-51096 Reims Cedex

Les Dialogues de Grégoire le Grand et leur postérité: une certaine idée de la réforme?

La réforme selon Grégoire le Grand, à la frontière entre l'Antiquité et le Moyen Âge, correspond à une volonté d'organisation et de correction, de remise en ordre, sans référence explicite ou régulière à un retour à une forme de communauté primitive idéale. Il s'agit davantage d'une remise en ordre morale du monde laïque, sur le modèle de ce monde monastique qui est, lui, le modèle très actuel dans lequel Grégoire s'est épanoui. L'hagiographie est bien propre à traiter ces aspirations, dont la radicalité est exprimée par la multiplicité et l'importance des miracles. Alors que leur abondance a longtemps jeté un doute sur la valeur des Dialogues, il conviendrait de considérer ces miracles plutôt comme les armes de Grégoire pour réformer les communautés chrétiennes. Le succès des Dialogues est d'emblée immense. Par l'étude de trois textes, qui s'inspirent directement de ceux-ci (les Vies des Pères de Mérida, la Passion de Praejectus) ou qui traitent sur un mode hagiographique de la figure de Grégoire (la première Vie de Grégoire le Grand), on peut voir comment ces aspirations à une réforme sont reprises, ou non, dans l'hagiographie du siècle suivant.

Grégoire le Grand - Dialogues - hagiographie - réforme - miracles.

\section{Gregory the Great's Dialogues and their Legacy : a Certain Idea of what a Reform can be?}

Reform, according to Gregory the Great (between Antiquity and the Middle Ages), is a mean to achieve organization, correction and restructuration, without having to make any explicit reference to the apostolic golden age of an ideal primitive community. It deals much more with a moral reform of the lay world, based on the model of the monastic world. That was indeed the very model in which Gregory found earlier on his full bloom. Hagiography is a very appropriate genre to express these aspirations, as well as the multiplicity and the importance of the miracles is a proper way to show their radicality. The Dialogues'authenticity has been for a long time under suspicion because of the many miracles it contains. But these should better be understood as Gregory's weapons to reform Christian 
communities. The Dialogues'success was at once a huge one. This study of three texts, either directly inspired by the Dialogues (Vitae sanctorum patrum Emeretensium, Vita Praeiecti) or dealing with Gregory'life itself (first Vita Gregorii), allows us to see whether the hagiography of the next century retains or not the aspirations to reform.

Gregory the Great - Dialogues - hagiography - reform - miracles. 
